\title{
Psicologia da saúde e produção científica'
}

\section{Health psychology and scientific output}

\author{
Geraldina Porto WITTER ${ }^{2,3}$
}

\begin{abstract}
Resumo
Este artigo é derivado de uma conferência que apresentou dois conceitos de saúde e algumas de suas implicações para a pesquisa e o trabalho de equipes de profissionais. Como exemplo, apresenta dados originais indicativos de que psicologia da saúde é uma área de pesquisa que apresenta bons indicadores de desenvolvimento nos estudos publicados, como título, autoria e volume de publicações em periódicos. Internacionalmente, não foram encontradas diferenças significativas entre estudos sobre saúde e sobre doença em uma amostra de 300 trabalhos publicados em revistas, livros e teses. No Brasil, a doença tende a ser o foco dos estudos. São também apresentadas considerações concernentes ao desenvolvimento da área e sobre a formação do psicólogo para atuar na área da saúde.
\end{abstract}

Unitermos: Cientometria. Formação profissional. Psicologia da saúde. Psicólogo.

\begin{abstract}
This paper derives from a conference that examined two concepts of health and some of its implications for research and the work of teams of professionals. As an example, it presents original data indicative of the fact that Health Psychology is an area of research that presents good indicators of development with the published studies, such as title, authorship, volume of publications in journals. Internationally, no significant differences between studies on health and disease were present in a sample of 300 papers published in journals, books and dissertations. In Brazil, the disease does tend to be the focus of studies. Considerations are also presented concerning the development of the area and about the professional training enabling the psychologist to work in the health area.
\end{abstract}

Uniterms: Scientometry. Professional development. Health psychology. Psychologist.

Há um contínuo no ciclo de vida que vai da concepção até a morte, embora em algumas áreas de conhecimento só se considere como ser o organismo nas etapas finais da gestação e, ainda assim, sob certas condições. Ao longo deste ciclo, a maioria dos seres humanos desfruta de saúde durante a maior parte do tempo, com episódios de doença. Alguns apresentam doenças crônicas que os levam precocemente ao final do ciclo: a morte. Tradicionalmente, o psicólogo trabalha ao longo deste ciclo; alguns enfocam prioritariamente a saúde, e outros, a doença.

A questão enfocada neste texto é analisar como a psicologia da saúde, especialmente em termos da produção científica, está se desenvolvendo. O tema

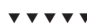

1 Artigo elaborado a partir da conferência apresentada na III Jornada de Psicologia da Saúde, da Faculdade de Medicina de São José do Rio Preto, em 2/6/2006

2 Universidade Camilo Castelo Branco, Pró-Reitoria de Pós-Graduação e Pesquisa, Coordenação da Pós-Graduação Stricto Sensu. R. Carolina Fonseca, 584, Itaquera, 08230-230, São Paulo, SP, Brasil.E-mails: <unicastelo@unicastelo.br>; <gwitter@uol.com.br>.

3 Universidade de São Paulo, Instituto de Psicologia. São Paulo, SP, Brasil. 
remete inicialmente ao que é saúde. Não se pode ignorar a história de vida das pessoas, da sociedade, o contexto sociocultural, as políticas de saúde, os custos etc.

Podem-se distinguir dois conjuntos de concepções sobre saúde (Epstein, 2005) e, com base em bibliografia recente, foi feita pela autora a elaboração da Figura 1.

O conceito A parte da clássica definição biopsicossocial da Organização Mundial de Saúde, que não vê saúde como mera ausência de doença. Acrescentase que, para tanto, não basta contar apenas com boa ciência para fornecer saúde a todos, é preciso ter condições para usá-la (Epstein, 2005; Ockene, 2006).

Um simples olhar para os dois conceitos mostra que suas características são distintas. $O$ conceito A é mais inclusivo: os aspectos psicológicos, sociais, ambientais e ecológicos são tão relevantes quanto os biológicos para determinar que uma pessoa desfruta de saúde. O conceito B baseia-se em exclusão: não ter doença é ter saúde, ainda que a pessoa não se considere saudável, seja infeliz, não desfrute de inclusão social etc.
O conceito A é muito amplo, genérico, comporta uma multiplicidade de perspectivas que, por um lado, constituem uma vantagem, por outro, trazem dificuldades para aplicação. Já o conceito B, por ser mais restritivo, é mais fácil de aplicar: se os exames clínicos e laboratoriais não indicam presença de doenças, falência ou falhas no funcionamento orgânico, trata-se de uma pessoa com saúde. Desta forma, para alguns profissionais é maior a aplicabilidade de $\mathrm{B}$ em relação ao conceito anterior.

Em decorrência das características já mencionadas, em A há uma maior consideração ou respeito à dimensão do que cada pessoa considera ter saúde, o que não é levado em consideração no conceito B, segundo o qual quem decide sobre a presença ou não de saúde é outro, não a própria pessoa, e esta será encaixada em um ou outro caso sem que sua opinião seja levada em consideração. No conceito A, o auto-relato da pessoa como saudável é valorizado em suas interações e no atendimento que lhe é oferecido (Hampson, Goldberg, Vogt \& Dubanosky, 2006). Em tais circunstâncias, pode-se dizer que o poder envolvendo
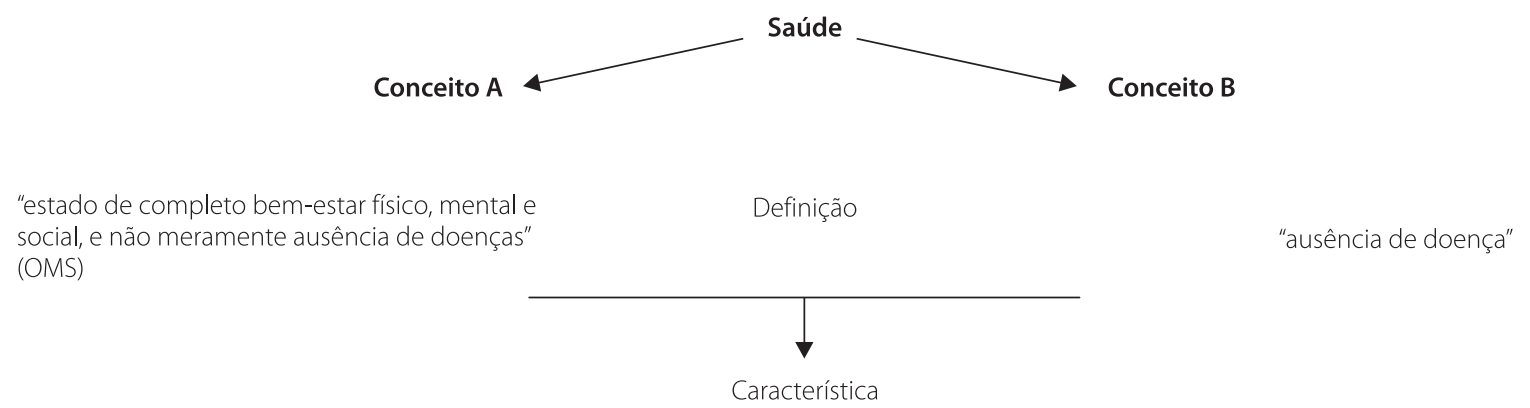

- inclusão

- Genérico

- Difícil aplicação

- Respeita a dimensão individual no que é "ter saúde"

- Poder distribuido

- Foco de atenção = pessoa

- Sistema centrado na pessoa

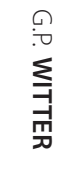

578

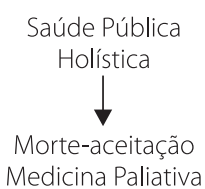

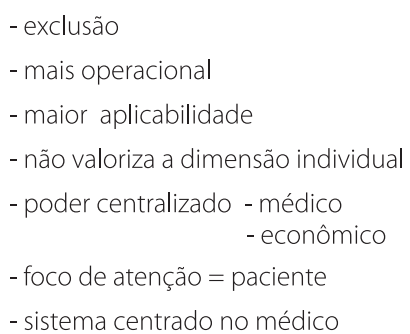

Ideário econômico

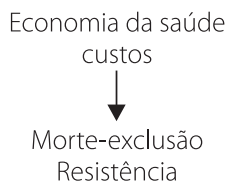


questões de saúde subjacente ao conceito A é distribuído ou partilhado por todos os envolvidos, e em B, esse poder está essencialmente nas mãos dos médicos e é influenciado por fatores econômicos e relações de custo-benefício.

Entretanto, pressões diversas têm atenuado um pouco a força do poder exclusivo do médico em questões de saúde. Há que se considerar o efeito do desenvolvimento técnico-científico de outras profissões que atuam na área da saúde e a conscientização da comunidade sobre a abertura trazida pela OMS na conceituação de saúde e dos direitos do consumidor em relação aos serviços. Não se pode esquecer que as questões de saúde tangem aspectos mais abrangentes do que as doenças e o aspecto financeiro. Como exemplo de resultados decorrentes das referidas pressões podem ser lembradas as preocupações mais recentes com as interações médico-paciente, a humanização da medicina e mesmo mudanças no código de ética desta área, para inserir aspectos pertinentes à matéria aqui enfocada (Epstein, 2005; Feitosa, 2001; Savioli, 2004).

No conceito A é a pessoa que constitui o foco da atenção, vista como um ser total e com suas relações sociais e culturais; no conceito $B$, a pessoa desaparece por trás do paciente, e a doença é que prevalece (Andrade, 1994).

Epstein (2005) lembra que ideários econômicos distintos estão subjacentes aos dois conceitos. No caso de A, a concepção econômica pode ser denominada como de saúde pública, caracterizada por busca de eqüidade e com uma perspectiva holística. Em B, o ideário denominado economia da saúde é mais pragmático e preocupado essencialmente com os custos. Um exemplo é a preocupação típica que aparece em Webster (2006) e trata da redução dos custos para assegurar a manutenção do grande negócio que se tornou a saúde.

As relações vida/saúde/doença/morte também divergem nos dois conceitos aqui considerados. Em A, trata-se de uma relação esperada. Embora a ênfase seja em vida-saúde, a morte é aceita como um fato natural e impossível de ser vencido. O que importa éa qualidade de vida, o quanto se sente bem a pessoa, o seu conforto biopsicológico. Nestas circunstâncias, a pessoa sem perspectiva de cura para sua doença, por exemplo, um paciente terminal, deve ser atendido dentro da moderna concepção de medicina paliativa, podendo mesmo se sentir saudável em sua vida social, psicológica e íntima. Já no caso do conceito $B$, a tendência é a negação da morte e o isolamento do paciente terminal, mesmo em relação aos seus familiares mais próximos, e a rejeição da medicina paliativa.

Em psicologia também são encontrados os dois conceitos, embora em alguns modelos, por sua própria origem e ênfase nos distúrbios, patologias e no tipo de serviço em que se envolvem, a força do conceito $B$ seja preponderante. A despeito do modelo assumido pelo profissional, parece haver um consenso sobre o fato de a psicologia ser prioridade para a saúde, como diz Arnett (2006). A forma como ela contribui para a saúde é que oscila: uns a restringem aos consultórios, clínicas e hospitais, outros dão a ela maior amplitude e apóiam-se mais nos modelos de desenvolvimento e de aprendizagem.

\section{Produção científica em psicologia da saúde}

Por séculos e, ainda hoje, a doença foi o foco da atenção dos pesquisadores (conceito B). Embora isto tenha sofrido algumas mudanças, esses pesquisadores ainda se concentram mais nos discursos do que na prática.

Parece oportuno lembrar que estudos com ênfase na saúde têm demonstrado, constantemente, os seus valores intrínsecos na busca do saber e como recurso para prever o futuro, como afirmam Hampson et al. (2006).

Na procura por uma perspectiva geral da relação pesquisa-saúde, foi feito pela autora um levantamento na base SciELO (16/3/2006). SciELO é uma base bibliográfica nacional, estatal, que cobre as publicações veiculadas nos principais periódicos brasileiros, incluindo todas as áreas do conhecimento. As próprias áreas por assunto disponíveis já evidenciam a tendência referida: foram encontrados 63 trabalhos, sendo mais freqüentes aqueles sobre psicopatologia ( $n=25$ ) e psicologia clínica $(n=15)$, e os demais, pouco freqüentes, conforme aparece na Tabela 1. Psicologia da saúde ficou em terceiro lugar, com o percentual de $11,11 \%$ da produção arrolada no SCiELO sobre saúde. As demais áreas constantes na base tiveram percentuais mais baixos de ocorrência e, assim, pouco relevantes para se detectar tendências gerais. De 
um modo geral, os dados por si só constituem uma evidência de que a preocupação predominante está voltada para a doença.

Como o foco deste trabalho foi a psicologia da saúde, destaca-se aqui toda a matéria levantada nesta área para uma síntese específica dos artigos registrados na SCIELO.

Torres (2003) apresenta um trabalho teórico em que enfoca as relações entre psicologia da saúde e bioética, tendo por substrato as questões de vida e morte. Lembra a crescente preocupação com a ética para a qual contribuem as revoluções tecnológica e biológica e o desenvolvimento social. A psicologia da saúde, por atuar em um contexto multidisciplinar desde o seu início, esteve envolvida com a ética, que define limites para a sua ação no ciclo vida-morte. Já Yamamoto e Cunha (1998) fazem uma caracterização dos psicólogos que atuam em hospitais não psiquiátricos na cidade de Natal, que reclamam do "desvio clínico do curso de graduação" e a insuficiente base para trabalhar na área. Yamamoto, Trindade e Oliveira (2002) apresentam um trabalho sobre psicólogos em hospitais no Rio Grande do Norte; na graduação, a maioria dos psicólogos parece não ter usufruído de oportunidade de aprender ou estagiar na área, mas alguns buscaram uma formação em pós-graduação.

Seidl (2005) estudou o enfrentamento em pessoas portadoras de HIV/AIDS ( $n=241$ ), levando em consi-

Tabela 1. Temas abordados em psicologia da saúde no PsycINFO (janeiro-abril de 2006).

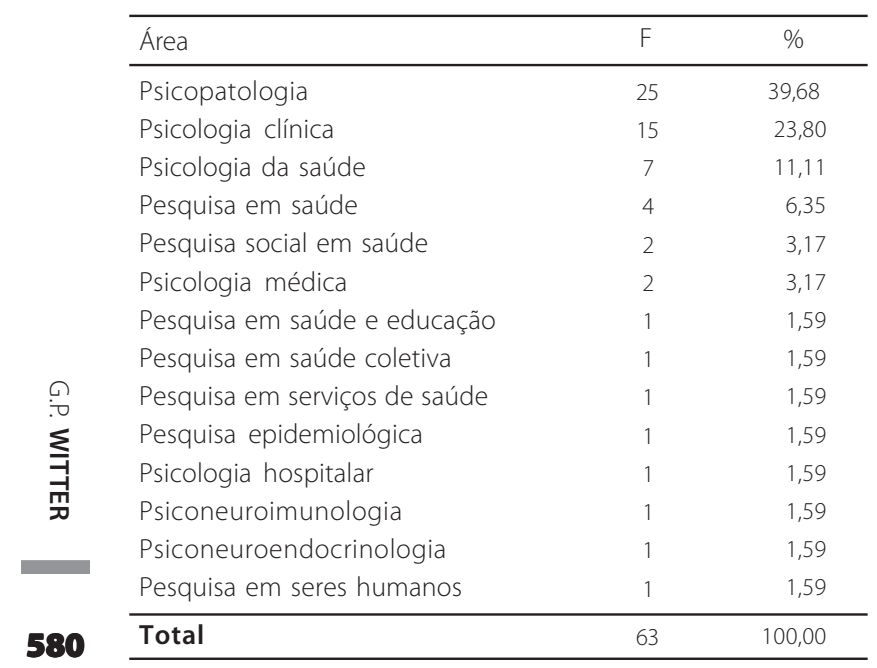

deração variáveis sociodemográficas, e encontrou variedade nas técnicas de enfrentamento, prevalecendo o foco na doença e não na saúde.

Miyazaki, Domingos, Valério, Santos e Rosa (2002) relatam o desenvolvimento do Serviço de Psicologia do Hospital de Base da Faculdade de Medicina de São José do Rio Preto (FAMERP) que, além do atendimento, implica em oferta de estágio e cursos. Kerbaury (2002) lembra a variedade de conceitos e denominações para a área de comportamento e saúde, mas afirma haver quase um consenso de que se deve enfocar a prevenção, o tratamento e ser multidisciplinar. Já Souza e Carvalho (2003) analisam o Programa da Saúde da Família, em pesquisa participante, no qual são atendidas aproximadamente 200 pessoas.

Em todos, o enfoque predominante é a perda da saúde, a doença, mesmo quando se trata de discutir aspectos mais subjetivos, como qualidade de vida. Assim, para uma perspectiva mais abrangente, foi realizada pela autora uma busca no PsycINFO, cobrindo o período de janeiro a abril de 2006, utilizando-se o termo Health Psychology como critério de pesquisa. A PsycINFO é a principal base bibliográfica sobre psicologia no mundo, gerenciada pela American Psychological Association.

Os resultados encontrados aparecem na Tabela 2. Das 27555 fontes registradas no período, 68,40\% eram artigos, $17,86 \%$ teses, 9,97\% livros ou capítulos de livros e $3,77 \%$ outros tipos de documentos. A alta concentração em periódicos $\left(\chi_{0}^{2}=105,83\right.$, n.g.l=3, $p \leq 0,05$, $\left.\chi_{c}^{2}=7,82\right)$ está dentro do esperado cientometricamente, permitindo concluir que a distribuição encontrada é indício de área bem desenvolvida (Población, Witter \& Silva, 2006).

Para verificar quais temas estão sendo enfocados pelos autores, optou-se por fazer uma amostra de cada tipo de suporte. Constituiu-se uma amostra sistemática de 100 referências de cada tipo, seguindo a ordem em que se apresentavam na base. Os resultados aparecem

Tabela 2. Psicologia da saúde no PsycINFO (Janeiro-abril de 2006).

\begin{tabular}{lrc}
\hline Tipo de documento & F & $\%$ \\
\hline Artigos & 18,848 & 68,40 \\
Teses & 4,920 & 17,86 \\
Livros/capítulos & 2,747 & 9,97 \\
Outros & 1,040 & 3,77 \\
\hline Total & 27,555 & 100,00 \\
\hline
\end{tabular}


na Tabela 3, totalizando 300 referências, suficientes para detectar as tendências temáticas de forma nítida.

No conjunto dos textos (Tabela 3), prevalece a categoria doenças/problemas psicológicos e sociais, com 30,33\%; a seguir, aparecem as práticas de saúde, com 24,00\%, e metodologia, com 10\%. Todavia, se aglutinadas algumas categorias, é a saúde (mais do que a doença) que prevalece: $6 \%$ dos trabalhos enfocaram a opinião de pessoas sobre o que é saúde, 2,67\% trataram da religiosidade e seu impacto na preservação e recuperação da saúde e 1,33\% estabeleceram condições para uma relação saudável entre sexualidade e saúde.

Vale destacar a preocupação com questões metodológicas. Guardadas as limitações da análise aqui realizada, o quadro internacional apresenta um equilíbrio entre o olhar prioritário à saúde, à doença e a aspectos mais neutros. Para testar esta possibilidade, foram recompostas as categorias e reagrupadas em: doença ( $n=91$ ), saúde (opinião + práticas + religiosidade + sexualidade $=102$ ) e neutra (história + legislação + mídia + metodologia + negócios + profissionais + serviços e programas $=107)$. $O$ teste de $\chi^{2}$ entre as três grandes categorias resultou em $\chi_{0}^{2}=29,57$ (Ho: $\chi^{2}=$ zero, n.g.l $=2, p \leq 0,05$ e $\chi_{c}^{2}=5,99$ ) (Siegel, 1956), ou seja, doença ficou abaixo do esperado, enquanto saúde e trabalhos neutros ficaram com freqüências superiores ao esperado, ocorrendo a rejeição de Ho. Para detalhar mais a análise, foi feito o teste de $\chi^{2}$ comparando-se estritamente as categorias doenças/problemas psicológicos e sociais ( $n=91$ ) com os totais das duas mais explicitamente associadas à saúde (práticas de saúde e perspectiva/opinião sobre saúde), que alcançaram $\mathrm{F}=90$. Neste caso, $\chi_{0}^{2}=$ zero, portanto menor que $\chi_{0}^{2}=3,84$

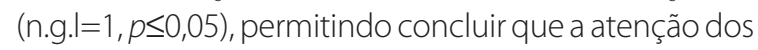
autores da base PsycINFO está distribuída de forma relativamente homogênea entre saúde e doença, possivelmente refletindo um crescimento do conceito $A$ de saúde. Aliás, este crescimento era esperado, face aos dados apresentados por Andrade (1994). Comparando com a base nacional, a situação é muito diferente; podese dizer que o conceito B ainda é mais forte no Brasil.

Um excelente exemplo desta perspectiva equilibrada de psicologia da saúde encontrada no exterior é o livro de Worell e Goodheart (2006), no qual saúde é o alicerce subjacente nos 50 capítulos, embora em vários deles a doença seja o foco principal, como no de Marsh (2006) sobre distúrbios emocionais e doenças mentais graves. A maioria trata mesmo é do desenvolvimento saudável e da preservação da saúde (Halper, 2006; Reynolds \& Repetti, 2006).

Autoria é uma característica psicométrica que tem merecido a atenção dos avaliadores da ciência (Población et al., 2006), esperando-se eqüidade de participação por gênero e predomínio de trabalhos em equipe. A análise das cem teses destacadas para estudo, por sua natureza, só contempla estudos de autoria única, mas permite a avaliação por gênero. Foram definidas as categorias masculino, feminino e indeterminada (quando não foi possível definir o gênero apenas com base no nome da pessoa). Por exemplo, o trabalho de Martin (2006) sobre relações de adaptação, perfeccionismo,

Tabela 3. Amostras $(n=100)$ de tipos de suporte da informação no PsycINFO (2006 até 19/5 (2006)): assuntos enfocados.

\begin{tabular}{|c|c|c|c|c|c|}
\hline Assunto & Livros & Testes & Artigos & Total & $\%$ \\
\hline Doenças/problemas psicológicos sociais & 21 & 44 & 26 & 91 & 30,33 \\
\hline História & 2 & & 6 & 8 & 2,67 \\
\hline Legislação & 8 & 4 & 1 & 13 & 4,33 \\
\hline Mídia e saúde & & & 9 & 9 & 3,00 \\
\hline Metodologia & 10 & 6 & 14 & 30 & 10,00 \\
\hline Negócios & 2 & 1 & 3 & 6 & 2,00 \\
\hline Perspectiva/opinião sobre saúde & 8 & 6 & 4 & 18 & 6,00 \\
\hline Práticas de saúde & 25 & 29 & 18 & 72 & 24,00 \\
\hline Profissionais & 14 & 5 & 3 & 22 & 7,33 \\
\hline Religiosidade & 3 & 1 & 4 & 8 & 2,67 \\
\hline Serviços/programas & 3 & 4 & 12 & 19 & 6,33 \\
\hline Sexualidade e saúde & 4 & & & 4 & 1,33 \\
\hline Total & 100 & 100 & 100 & 300 & 99,99 \\
\hline
\end{tabular}


estresse e ajustamento psicológico registrou-se em masculino; a pesquisa de Helfritz (2006) sobre agressividade foi para a categoria feminina, e o trabalho de Abdullah-Al-Mamun (2006) sobre cigarro e adição, taxação e saúde foi marcado na categoria indeterminado. Verificou-se que 30\% dos trabalhos eram de autoria masculina e $47 \%$ feminina, ficando os indeterminados com $23 \%$. O teste $\chi^{2}$ entre os gêneros masculino e feminino resultou em 3,75, portanto, inferior ao $\chi_{c}^{2}=3,84(p \leq 0,05$, n.g.l. $=1)$. Pode-se inferir que embora mais mulheres tenham defendido teses do que o fizeram os homens, estatisticamente não houve diferença entre eles.

Outro indicador forte de desenvolvimento de uma área científica é o predomínio de trabalhos realizados em equipe, ou seja, publicações com autoria múltipla (dois ou mais autores) em lugar de trabalhos individuais. Para verificar este preditor de desenvolvimento na área, foi analisada a autoria nas cem referências de artigos de periódico do PsycINFO. Encontrou-se $4 \%$ de trabalhos para os quais não constou autor (No authorship indicated), como no texto One hundred years ago: asylum report (2006). Foram assinados por mais de um autor 69\% dos trabalhos. Um exemplo é o de Kashi e Ganesh (2006), que trata de uma questão cada vez mais presente na área: a relação entre espiritualidade/ religiosidade e saúde. Os restantes (27\%) foram assinados por uma única pessoa, como o trabalho de King (2006) sobre preparo de psicólogos e da psicologia para os novos tempos na área da saúde. Para verificar se havia diferença estatística entre a ocorrência de trabalhos de autoria múltipla e única foi feito o teste $\chi^{2}$, resultando em $18,36\left(\chi_{c}^{2}=3,84, p \leq 0,05\right)$, o que permite concluir que é estatisticamente significativa a diferença. Vale dizer que houve grande ocorrência de trabalhos em autoria múltipla, um forte indicador de bom desenvolvimento da área, mas a formação de equipes ainda precisa ser mais estimulada.

Outro aspecto importante de um trabalho científico é o seu título, já que é o primeiro contato do leitor com o texto e tem suas palavras substanciais usadas para registro nas bases de dados. Deve ser claro, preciso e sucinto. Espera-se que tenha até 12 vocábulos, embora às vezes as bases, periódicos e eventos restrinjam até 10. Também é considerado um dado que serve de indicador de desenvolvimento científico da área respeitar estes limites (Población et al., 2006). No caso das teses aqui enfocadas, o registro foi de $42 \%$ com os títulos dentro das dimensões esperadas, e 58\% as que excederam. O teste de significância resultou em $\chi_{c}^{2}=2,56$, inferior ao valor crítico $\left(\boldsymbol{\chi}_{c}^{2}=3,84, p \leq 0,05\right.$, n.g.l=1). Vale dizer que há eqüidade estatística entre o número de trabalhos dentro e fora do esperado. Pode-se dizer que há necessidade de maior criticidade, criatividade e cuidado na elaboração dos títulos dos trabalhos na área de psicologia da saúde para que alcancem padrões já atingidos em outras áreas da psicologia (Buriti, 2003; Carelli, 2002; Pacheco, 2003).

Os dados apresentados mostram desenvolvimento diferenciado no Brasil e no exterior, mas as perspectivas gerais são positivas. Parece oportuno tecer algumas considerações sobre o futuro na área, o que é feito a seguir.

A psicologia da saúde é uma área que vem crescendo rapidamente em nível nacional e internacional, como se pode deduzir do número de trabalhos indexados na base PsycINFO. Entretanto, há necessidade de muitas melhorias no setor, tanto técnico-científicas quanto culturais, sendo as últimas mais lentas e difíceis de conseguir.

O primeiro aspecto trata da concepção de saúde mantida pela população em geral e pelos profissionais da área, pelos governos e mesmo pelos sistemas de saúde e empresas de seguro-saúde, como aparece em Romanow (2006). Há necessidade de mais pesquisas na área, de um amplo debate e busca de bases consensuais mais válidas para definir os vários aspectos envolvidos.

O segundo aspecto diz respeito às mudanças nos sistemas de saúde-doença, seja na dimensão econômica, na inserção dos vários tipos de profissionais da área ou na ênfase em tópicos diversos. Mudanças na área são prementes e envolvem muitas questões além da financeira. Há um certo comodismo na manutenção do estado vigente, embora as críticas sejam freqüentes.

O terceiro aspecto enfoca as mudanças na própria psicologia, desde a sua concepção, passando por uma revisão de sua estruturação em áreas, particularmente da saúde. Há necessidade de rever suas ênfases temáticas, de olhar o ser humano globalmente e não apenas como portador de problemas biopsicossociais. Ao homem devem-se oferecer programas de prevenção, além dos de remediação. No Brasil tem-se privilegiado o segundo caso, o que requer correção. Essas mudanças 
precisam ser objeto de consideração e discussão no âmbito das universidades.

O quarto aspecto decorre do anterior e é básico para qualquer mudança. Considerando que muitas das mudanças precisam primeiramente ocorrer na universidade, quer em termos de pesquisas, quer de formação de recursos humanos, é preciso que essas instituições revejam o que estão oferecendo em termos de saúde, como bem lembra McDermott (2005).

No Brasil, há premente necessidade de mudanças curriculares de cunho técnico-científico na formação do psicólogo, para que este profissional possa construir seus planos de ação junto a colegas de outras áreas. Como lembra Johnson (2003), há necessidade de o psicólogo desenvolver competências para atuar em saúde com pessoas que se consideram saudáveis. Também precisa saber trabalhar com pessoas com doenças diversas. O treino para atuação clínica psicológica é insuficiente, com sérios conflitos decorrentes, quando esta atuação é transportada para setores como hospitais, atendimento a doentes crônicos, empresas etc. Deve haver equilíbrio na formação entre saúde e doença (Yamamoto \& Cunha, 1998; Yamamoto et al., 2002), com ensino eficiente e atual (Hill IV, 2006).

Precisa ser revista também a formação oferecida pelos cursos de doutorado (Arnett, 2006) que, muitas vezes, acabam por hiperdimensionar a doença, sem dar relevância ao conhecimento do que seja o "normal". O autor refere-se à formação no Canadá, que ele considera relativamente limitada em questões de saúde, sofrendo deformação em decorrência da ênfase clínica de muitos programas que refletem o predomínio do regime de privilegiar a doença sobre o desenvolvimento de um real sistema de saúde, mantendo a fragmentação dos serviços de saúde física e mental. Além disso, tanto na graduação como na pós-graduação, a prática da interdisciplinaridade deve ser uma constante, segundo Kerbaury (2002).

O quinto aspecto diz respeito às prioridades de pesquisa. Tendo por base alguns autores já referidos, é prioritário conhecer a saúde e o que caracteriza as pessoas saudáveis. Arnett (2006) considera que o fato de o governo canadense encaixar a psicologia como disciplina da saúde não está correto, pois a maioria dos profissionais da área pouco sabe sobre saúde e sistema de saúde. Pouco podem contribuir, devido à sua forma- ção de olhar essencialmente clínico, para melhorar o sistema de saúde. Para serem efetivos, precisariam pesquisar mais sobre saúde, novas tecnologias e desenvolver "uma vasta gama de atividades ligadas ao domínio da saúde" (p.32).

Podem-se acrescentar aqui as considerações de Chalizk (2006), que afirma que para haver progresso substancial na área é necessário capacidade para saber usar as evidências empíricas, com base em dados estatísticos, para sustentar a prática. Além de novas pesquisas, é necessário fazer revisões usando a metodologia da meta-análise. Entretanto, é preciso lembrar que, como diz Ockene (2006), ao se referir especificamente à medicina comportamental, não basta para desenvolver a saúde contar com uma contribuição científica persistente, se não houver grande efeito desta na saúde pública.

\section{Considerações Finais}

Convivem na atualidade dois conceitos de saúde, embora a mistura de partes deles possa ocorrer. Isto se reflete no atendimento, nos programas e nas pesquisas, já que eles estão explícita ou implicitamente presentes na ação e no direcionamento da busca de conhecimentos na área. Embora a produção no exterior seja rica e enfoque tanto saúde como doença, no Brasil ela ainda é tímida e tende a olhar mais para a doença.

De um modo geral, pode-se dizer que a área está em desenvolvimento, com alguns indicadores promissores, mas há muito por fazer para que alcance patamares mais elevados. Isto requer que sejam revistos seus objetivos, conceitos, referenciais conceituais, a formação do psicólogo e a reestruturação da própria psicologia em termos de áreas e sub-áreas. Há muito por pesquisar e discutir para que os avanços científicos realmente contribuam para mudar a realidade. Os psicólogos podem fazer muito no setor e devem fazê-lo baseados em uma visão multidisciplinar.

\section{Referências}

Andrade, C. R. F. (1994). Fonoaudiologia preventiva: discursos científico-pedagógico, modelo teórico e vocabulário técnico-científico. Tese de doutorado não-publicada, Universidade de São Paulo.

Abdullah-Al-Mamun, K. S. (2006). Three essays on cigarret addiction, taxation and health. Dissertation Abstract 
International: section A. Humanities and Social Sciences, 66 (7A), 2654

Arnett, J. L. (2006). La psychologie et la santé. Canadian Psychology, 47 (1), 33-35.

Buriti, M. S. L. (2003). Psicologia do esporte: análise da produção científica via periódicos. Tese de doutorado não-publicada, Pontifícia Universidade Católica de Campinas.

Carelli, A. E. (2002). Produção científica em leitura: dissertações e teses (1990-1999). Tese de doutorado não-publicada, Pontifícia Universidade Católica de Campinas.

Epstein, T. (Org.). (2005). A comunicação também cura na relação entre médico e paciente. São Paulo: Angellara.

Feitosa, L. (2001). Humanização nos hospitais. Fortaleza: Premius.

Halpen, D. F. (2006). Girls and academic success: changing patterns of academic achievement. In J. Worrel \& C. D. Good Heart (Orgs.), Handbook of girl's and women's psychological health (pp.272-282). London: Oxford University Press.

Hampson, S. E., Goldberg, L. R., Vogt, T., \& Dubanoski, J. P. (2006). Forty years on: teacher's assessments of childeren's personality traits predict self-reported helth behaviors and outcomes in middle life. Health Psychology, 25 (1), 57-64.

Helfritz, L. E. (2006). Looking for trouble? Processing of threat in impulsive and premeditated aggression: an event-related potential study. Dissertations Abstracts International: Section B: The Sciences and Engineering, 66 (7B), 3990

Hill IV, G. W. (2006). The society for the teaching of psychology: a psychology teacher's best friend. In W. Buskit \& S. F. Davis (Orgs.), Handbook of the teaching of psychology (pp.23-28). Malden: Blackwell.

Johson, N. G. (2003). A step in the right direction: commenton Belar et al (2001) Prevention and treatment. Retrieved October 22, 2005, available from http:// journals.apa.org/prevention/volume 6/pre00628c.html

Kashi, K., \& Ganesh, L. S. (2006). Spirituality in health care organizations. Journal of the Indian Academy os Applied Psychology, 32 (2), 159-167.

Kerbaury, R. R. (2002). Comportamento e saúde: doenças e desafios. Psicologia USP, 13 (1), 11-28.

King, M. C. (2006). Preparing psychology and psychologists for new health care markets. Canadian Psychology, 47 (1), 51-56.

Marsh, D. T. (2006). Serious emotional disturbance and serious mental illness. In J. Worell \& C. D. Good Heart, (Orgs.), Handbook of girls 'and women's psychological health. Oxford: Oxford University Press.

Martin, J. L. (2006). Relations between adaptive and maladaptive perfectionism, stress, and psychological adjustment. Dissertation Abstract International: Section B: The Sciences and Engineering, 66 (7B), 3.989.

McDermott, R. J. (2005). Inside the academy. American Journal of Health Behavior, 30 (2), 221-222.
Miyazaki, M. C. O. S., Domingos, N. A. M., Valério, N. I., Santos, A. R. R., \& Rosa, L. T. B. (2002). Psicologia da saúde: extensão de serviços à comunidade, ensino e pesquisa. Psicologia USP, 13 (1), 29-53.

Ockene, J. K. (2006). Fulfilling our assignment to improve the health ofall: good science just isn't enough. Annals of Behavior Medicine, 31 (1), 14-20.

One hundred years ago: asylum report (2006). British Journal of Psychiatry, 188 (1), 90.

Pacheco, E. M. C. (2003). Avaliação psicológica: avaliação de periódicos. Tese de doutorado não-publicada, Pontifícia Universidade Católica de Campinas.

Poblacion, D. A., Witter, G. P., \& Silva, J. F. M. (Orgs.) (2006). Comunicação \& produção científica:contexto, indicadorese avaliação. São Paulo: Angellara.

Reynolds, B. M., \& Repetti, R. L. (2006). Adolescent girls'health in the context of peer and community relationships. In J. Worell \& C. D. Good Heart (Orgs.), Handbook of girls 'and women's psychological health (pp.292-300). Oxford: Oxford University Press.

Romanow, R. J. (2006). Le régie d'assurance-maladie du Canadá-Arrive-t-il à un tournant? Canadian Psychology, 47 (1), 9-18

Savioli, R. M. (2004). Milagres que a medicina não contou. São Paulo: Gaia.

Seidl, E. M. F. (2005). Enfrentamento, aspectos clínicos e sociodemográficos de pessoas vivendo com HIV/AIDS. Psicologia em Estudo, 10 (3), 421-429.

Siegel, S. (1956). Non parametric statistics for the behavioral Sciences. New York: McGraw Hill Book Co.

Souza, R. A. \& Carvalho, A. M. (2003). Programa de saúde da família e qualidade de vida: um olhar da Psicologia. Estudos de Psicologia, 8 (3), 515-523.

Torres, W. C. (2003). A bioética e a psicologia da saúde: reflexões sobre questões de vida e morte. Psicologia: Reflexão e Crítica, 16 (3), 475-482.

Webster, P. (2006). US big businesses struggle to cope with health-care costs. Lancet, 367 (9505), 101-102.

Yamamoto, O. H., \& Cunha, I. M. F. F. O. (1998). O psicólogo em hospitais de Natal: uma caracterização preliminar. Psicologia: Reflexão e Crítica, 11 (2), 345-362.

Yamamoto, O. H., Trindade L. C. B. O., \& Oliveira T. S. (2002). O psicólogo em hospitais no Rio Grande do Norte. Psicologia-USP, 13 (1), 217-246.

Webster, P. (2006). US big businesses struggle to cope with health: care costs. Lancet, 365 (9505), 101-102.

Worell, J., \& Goodheart, C. D. (Orgs.). (2006). Handbook of girls' and women's psychological health. Oxford: Oxford University Press.

Recebido em: 23/10/2006

Versão final reapresentada em: 13/4/2007

Aprovado em: 22/5/2007 